\title{
The Comparison of Personality Profiles of Sports and Non-sports Group by Six-factor Model of Personality
}

\author{
Aylin Zekioglu ${ }^{1, *}$, Arkun Tatar², Hudanur Ozdemir ${ }^{2}$ \\ ${ }^{1}$ Sports Science Faculty, Manisa Celal Bayar University, Manisa, Turkey \\ ${ }^{2}$ Department of Istanbul, Faculty of Literature Psychology, Fatih Sultan Mehmet Vakif (FSM) University, Turkey
}

Copyright $(\mathrm{C} 2018$ by authors, all rights reserved. Authors agree that this article remains permanently open access under the terms of the Creative Commons Attribution License 4.0 International License

\begin{abstract}
Since the HEXACO framework of personality is relatively newer than other models, the number of studies conducted relying on this model is fewer respectively. For this reason, the purpose of the present study is to examine the sports participation and personality relation in non-athlete adults based on the above-mentioned personality model and to compare personality profiles of the individuals who do and who do not engage in sports. The people who agreed to take part in this study were 1013 people in total, 537 of them were female and 476 were male. The main effect in the whole group was statistically significant. When the result is considered for the factors separately, statistically significant differences were detected among the groups for the Honesty-Humility factor, for the Emotionality factor, for the Extraversion factor and for the Openness to Experience factor. The Main Effect was statistically significant in the female group. Statistically significant differences were determined among the groups for the Emotionality factor, for the Extraversion factor, for the Conscientiousness factor and for the Openness to Experience factor. When only the male group was considered, it was determined that the Main Effect was statistically significant. Statistically significant differences were detected among the groups for the Emotionality factor, for the Extraversion factor and for the Openness to Experience factor. When the study is evaluated in general terms, it is seen and can be said that there is a relation between doing sports and personality traits in a Turkish-speaking participant group.
\end{abstract}

Keywords Sports, Non-athlete, Six-factor Model of Personality, HEXACO

\section{Introduction}

Conceptualization of individual differences and the classification of personality traits has been constituted one of the main problems of personality psychology. Most researchers suggest that identifying descriptive words (adjectives) which define and are representatives of personality characteristics in a language through lexical studies, and grouping them with the use of the factor analysis is a viable method to solve the problem $[1,2,3]$. Following this approach, $[4,5,6]$ has recently proposed a six-dimensional model (HEXACO) which was derived from lexical studies that were conducted in many different languages and cultures. It is claimed that the model is more comprehensive and stronger compared to other models such as the Big Five or the Five Factor Model, noting that HEXACO contains additional variables and its validity has been demonstrated in various languages [7, 8, 9, 10]. Besides forming an acronym for the factors, HEXACO also reflects the number of factors.

There are six factors defined in the model, which are Humility-Honesty (H), Emotionality (E), Extraversion (X), Agreeableness (A), Conscientiousness (C), and Openness to Experience $(\mathrm{O})[8,11]$. Based on this model, the HEXACO Personality Inventory was developed by the researchers, containing and assessing aforementioned factors $[12,13,14]$. The Humility-Honesty Factor, which is claimed to be the most important factor of the HEXACO, as it contains additional personality characteristics that differentiate the proposed model from other models, consists of Sincerity, Fairness, Greed-Avoidance, and Modesty facets $[15,16,17]$, It is stated that while Fairness and Sincerity facets refer to "honesty", the Modesty and Greed-Avoidance facets refer to "humility" [6]. Individuals who receive high scores from this factor, as it is reported, tend to be sincere, honest, loyal, humble and fair, therefore they generally avoid manipulating and cheating other people [13]. In addition, it was also reported that "Humility-Honesty" and "Agreeableness" factors refer to "reciprocal altruism" tendency together by completing each other [6].

Emotionality is the second factor in the model and it consists of Fearfulness, Anxiety, Dependence, and Sentimentality facets [14]. It was reported that 
Emotionality factor overlaps with Neuroticism / (low) Emotional Stability factor from the five factor framework at a great deal; however, considering that the Neuroticism and Emotional Instability concepts may be humiliating or "pejorative", this factor was named as "Emotionality" instead [9,11]. In addition, it was reported that emotionality factor, differently from the Neuroticism factor, does not contain hostile feelings and emotional temperamental $[6,11,12]$. The Fearfulness, Anxiety, Dependency and Sensitivity facets in the factor measure the tendency of being fearful, anxious, and hypersensitive at one end, and being independent, self-Confident, strong and brave in the other end. It was stated that individuals receiving high scores in this factor are more sensitive especially to physical pain, and show the tendency of experiencing anxiety and stress in diverse situations [11, 13]. In addition, it was also stated that individuals who are at the high end of this factor establish strong bonds with people, and expect intense interest and support from the people with whom they develop dependency in situations that cause anxiety [13].

Extraversion, which is the third factor, is evaluated with the Expressiveness, Social Boldness, Sociability and Liveliness facets [14]. It was reported that the Extraversion factor, overlaps with the Extraversion factor defined in the Big Five personality model in terms of characteristics such as talkativeness, sociability, activeness, cheerfulness, at one end, and shyness, passiveness, quietness at the other. On the other hand, it was reported that in the HEXACO model, features like braveness, strength, self-confidence are recovered at the positive end of the Emotionality factor rather than the Extraversion factor, unlike the Extraversion factor within the Big Five $[6,12,13]$. Individuals who receive high scores in this factor are more likely to feel comfortable and confident in various social situations, have an enthusiastic and lively speech, enjoy social interactions; however, individuals with low scores have difficulty when speaking in public, and prefer to be alone instead of being in social settings [13]. The Agreeableness factor, which is the fourth factor, includes Forgiveness, Gentleness, Flexibility and Patience facets [13]. Individuals who are at the high end of this factor are defined as patient, tolerant, peaceful, easy-going/mild, agreeable and kind; and individuals who are at the low end are defined as ill-tempered, moody, aggressive, stubborn and angry [11]. In addition, it was stated that agreeable people tend to trust and continue a relationship even if they are treated badly, yet incompatible people tend to judge other people ruthlessly and have grudge [13]. It was also stated that the HEXACO Agreeableness doesn't contain over emotionality like the Big Five Agreeableness, and also the emotional temperamental phenomenon is recovered in the low/negative end of this factor $[6,11]$. The Conscientiousness Factor, which is the fifth factor, is evaluated with the Organization, Diligence, Perfectionism and Prudence facets [14]. It was reported that the individuals in the high end of the Conscientiousness factor, who are defined as organized, diligent, cautious and meticulous, tend to seek order in their physical environment, have high motivation, are more successful in structured missions, and are strictly devoted to work ethics. It was also stated that the individuals in the low end of this factor, who are defined as messy, negligent, indifferent, lazy, irresponsible, absent-minded, and forgetful, tend to live haphazardly, make impulsive decisions, and act without a plan and without thinking the consequences. In addition, it was also stated that the motivation and self-discipline of such individuals for success found to be low $[11,13]$.

The Openness to Experience Factor, which is the last factor in the model, consists of Aesthetic Appreciation, Inquisitiveness, Creativity and Unconventionality facets [14]. It was stated that the individuals in the high end (which is defined with the intellectual, enlightened, creative, unconventional, innovative and ironic personality traits) enjoy art, natural beauties, and traveling, and tend to express themselves through art and are open-minded. It was also stated that individuals in the low end (which is defined with the shallow, superficial, uncreative, weak imagination and traditional personality traits) do not have interest in social sciences and art; are not open to extraordinary viewpoints and cannot produce original ideas $[11,13]$.

In addition, there is another dimension called Altruism versus Antagonism in the inventory. It was stated that this dimension was not a separate factor but functioned as a "filling dimension" $[11,18,19]$.

It was reported that individuals who receive high scores in the end that represents humility-honesty, agreeableness and emotionality factors have the tendency of being softhearted and understanding, as they try to avoid damaging weak people and show a generous attitude to such people. It was also stated that individuals at the low end might be cold-hearted to people who are damaged by something or somebody, and in such situations, they may show insensitive attitudes towards such people instead of feeling sorry for them [11].

Since the HEXACO framework of personality is relatively newer than other models, the number of studies conducted relying on this model is fewer respectively. As the HEXACO Personality Inventory was translated into Turkish recently [18], there are no studies conducted in Turkish-speaking samples based on this model. For this reason, the purpose of the present study is to examine the sports participation and personality relation in non-athlete adults based on the above-mentioned personality model and to compare personality profiles of the individuals who do and who do not engage in sports. In this context, answers will be sought for three questions. The first question is whether the personality traits of the individuals who frequently engage in sports, who occasionally engage in sports or who do not engage in sports show differences according to the HEXACO personality model; if these groups differ from each other, then the second question is 
in which characteristics they show differences. Finally the last question of the present study is whether there are gender differences in terms of the answers given for the first two questions. In the light of these explanations, the purpose of the study was to examine the personality profiles in terms of doing or not doing sports. The differences between those who did and who did not do sports were determined with a descriptive approach in line with the HEXACO Model.

\section{Materials and Methods}

\subsection{Participants}

The people who agreed to take part in this study were 1013 people in total, 537 of them were female $(53.0 \%)$ and 476 were male $(47.0 \%)$. The ages of the participants varied between 16 and 75 years (mean $=36.14 \pm 11.23$ years). 124 $(12.2 \%)$ of the participants stated that they regularly do sports, $569(56.2 \%)$ stated that they occasionally do sports, and $320(31.6 \%)$ stated that they do not do sports at all. 118 $(11.6 \%)$ of the participants were elementary and middle school graduates, 275 (27.1\%) were high school graduates, and $620(61.2 \%)$ were university students or graduates. When asked about their marital status, $471(46.5 \%)$ of participants stated that they are single, 493 (48.7\%) stated that they are married, and $49(4.9 \%)$ stated that they are widowed or divorced. The income status of $49(4.8 \%)$ people was low-bad, $676(66.7 \%)$ people was medium and 288 (28.4\%) people was high-good. Being an athlete or sports professional was taken as the exclusion criterion, thus professional sports-doers (athletes) or those who worked in sportive settings were not included in the present study.

\subsection{Material}

The Turkish form of the Revised HEXACO Personality Inventory [18] and a brief socio-demographic questionnaire form were used in the present study. The HEXACO Personality Inventory consists of 24 facets under six-factors designed as second order, in the form of 5 -Point Likert Type Scale $(5=\mathrm{I}$ definitely agree, $1=\mathrm{I}$ definitely do not agree). The revised full form of the inventory includes 100 items $[13,14,19]$ and the inventory has also a short form including 60 items [12,19]. The Inventory was translated into many languages as well as Turkish, and the Turkish form was investigated in terms of its psychometric features [18].

\subsection{Procedure}

The present study was conducted with people who could be contacted using the convenient sampling method. The inventory was applied individually and one single application lasted approximately 30-40 minutes. The whole process lasted six months.

\subsection{Data Analysis}

First of all, reliability analyses of the HEXACO Personality Inventory was made. Then, the total scores of the HEXACO Personality Inventory's factors and facets were compared in terms of sports frequency in both for male and female groups separately with the Multivariate Analysis of Variance (MANOVA).

\subsection{Results}

According to the results, the internal consistency coefficients varied between 0.72 and 0.77 for the six-factors of the HEXACO Personality Inventory in the whole group (Table 1).

After determining the reliability coefficients, for each six factor, total scores of the whole group, the female, and the male group were compared in terms of their sports status with the MANOVA. According to the results, the main effect in the whole group was statistically significant; Wilks Lamda $=0.91, \mathrm{~F}(12,2010)=8.25, \mathrm{p}<0.001$, Eta Squared $=0.05$. When the result is considered for the factors separately, statistically significant differences were detected among the groups for the Honesty-Humility factor $(\mathrm{F}(2,1010)=4.10, \mathrm{p}<0.05$, Eta Squared $=0.01)$, for the Emotionality factor $(\mathrm{F}(2,1010)=20.68, \mathrm{p}<0.001$, Eta Squared $=0.04)$, for the Extraversion factor $(F(2,1010)=$ $19.84, \mathrm{p}<0.001$, Eta Squared $=0.04)$ and for the Openness to Experience factor $(\mathrm{F}(2,1010)=18.69, \mathrm{p}<0.001$, Eta Squared $=0.04)$. According to the results of the Tukey post-hoc test, it was determined that the group doing sports regularly had a higher mean score than the other two groups in the Honesty-Humility factor. There is also a difference between the three groups in the Emotionality Factor. The group doing sports regularly had a higher mean score than the other two groups, and the group that do sports occasionally had a higher mean score than the group that does not do sports at all. 
Table 1. The HEXACO Personality Inventory internal consistency coefficients and the MANOVA results of the groups that were formed according to the sports status

\begin{tabular}{|c|c|c|c|c|c|c|c|}
\hline $\begin{array}{l}\text { Dependent Variable } \\
\text { (Domain) }\end{array}$ & $\begin{array}{l}\text { Cronbach } \\
\text { Alpha }\end{array}$ & $\begin{array}{c}\mathrm{F} \\
\text { (All Group) }\end{array}$ & $\eta^{2}$ & $\begin{array}{c}\mathrm{F} \\
\text { (Female) }\end{array}$ & $\eta^{2}$ & $\begin{array}{c}\text { F } \\
\text { (Male) }\end{array}$ & $\eta^{2}$ \\
\hline Main effect & & $8.25 * * *$ & 0.05 & $4.17 * * *$ & 0.05 & $2.88 * * *$ & 0.04 \\
\hline Honesty-Humility & 0.76 & $4.10^{*}$ & 0.01 & 1.38 & 0.01 & 2.02 & 0.01 \\
\hline Emotionality & 0.72 & $20.68 * * *$ & 0.04 & $4.63 * *$ & 0.02 & $5.46 * *$ & 0.02 \\
\hline Extraversion & 0.77 & $19.84 * * *$ & 0.04 & $9.81 * * *$ & 0.04 & $10.19 * * *$ & 0.04 \\
\hline Agreeableness & 0.73 & 1.28 & 0.00 & 2.05 & 0.01 & 0.29 & 0.00 \\
\hline Conscientiousness & 0.76 & 2.34 & 0.01 & $3.44 *$ & 0.01 & 0.78 & 0.00 \\
\hline Openness to Experience & 0.74 & $18.69 * * *$ & 0.04 & $13.40 * * *$ & 0.05 & $4.80 * *$ & 0.02 \\
\hline
\end{tabular}

There was also significant differences between the three groups in the Extraversion factor as well. However, the group doing regular sports had the lowest mean scores, and the group that does sports occasionally had lower mean scores than the group that does not do sports at all. The group that does not do any sports had the highest mean scores in the Openness to Experience factor. No statistically significant differences were detected for the Agreeableness factor $(\mathrm{F}(2,1010)=1.28, \mathrm{p}>0.05$, Eta Squared $=0.00)$ and Conscientiousness factor $(F(2,1010)$ $=2.34, \mathrm{p}>0.05$, Eta Squared $=0.01)$ among the three groups (Table 1, Figure 1)

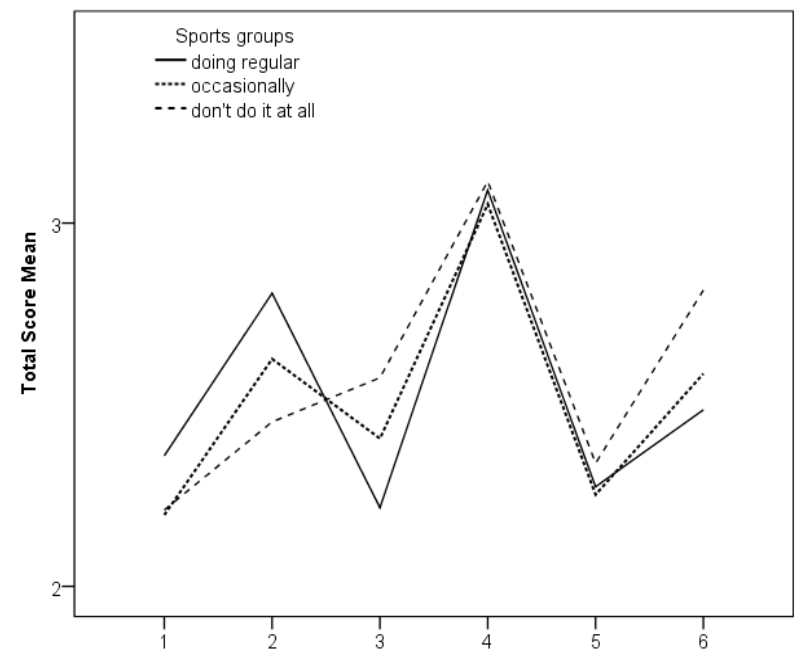

1: Honesty-Humility Domain, 2: Emotionality Domain, 3: Extraversion Domain, 4: Agreeableness Domain, 5: Conscientiousness Domain, 6: Openness to Experience Domain

Figure 1. Comparison of the total scores of the groups that were formed according to the sports status in the general group

The Main Effect was statistically significant in the female group; Wilks Lamda $=0.91, \mathrm{~F}(12,1058)=4.17$, $\mathrm{p}<0.001$, Eta Squared $=0.05$. Statistically significant differences were determined among the groups for the Emotionality factor $(\mathrm{F}(2,534)=4.63, \mathrm{p}<0.01$, Eta Squared $=0.02)$, for the Extraversion factor $(\mathrm{F}(2,534)=9.81$, $\mathrm{p}<0.001$, Eta Squared $=0.04)$, for the Conscientiousness factor $(\mathrm{F}(2,534)=3.44, \mathrm{p}<0.5$, Eta Squared $=0.01)$ and for the Openness to Experience factor $(\mathrm{F}(2,534)=13.40$, $\mathrm{p}<0.001$, Eta Squared $=0.05$ ). According to the result of the Tukey post-hoc test, those who do not do any sports had lower scores than those who do sports occasionally in the Emotionality factor. In the Conscientiousness factor, those who do not do any sports had higher average scores than those who do sports occasionally. There was also a significant difference between the three groups in the Extraversion factor; however, the group that does sports regularly had lower mean scores than the other two groups, and the group that occasionally does sports had lower mean scores than the group that does not do any sports. In the Openness to Experience factor, the group that does sports regularly and the one that occasionally does sports had lower mean values than the group that does not do any sports at all. No significant differences were detected among the three groups for the Honesty-Humility factor (F $(2,534)=1.38, p>0.05$, Eta Squared $=0.01)$, and for the Agreeableness factor $(\mathrm{F}(2,534)=2.05, \mathrm{p}>0.05$, Eta Squared $=0.01)($ Table 1, Figure 2).

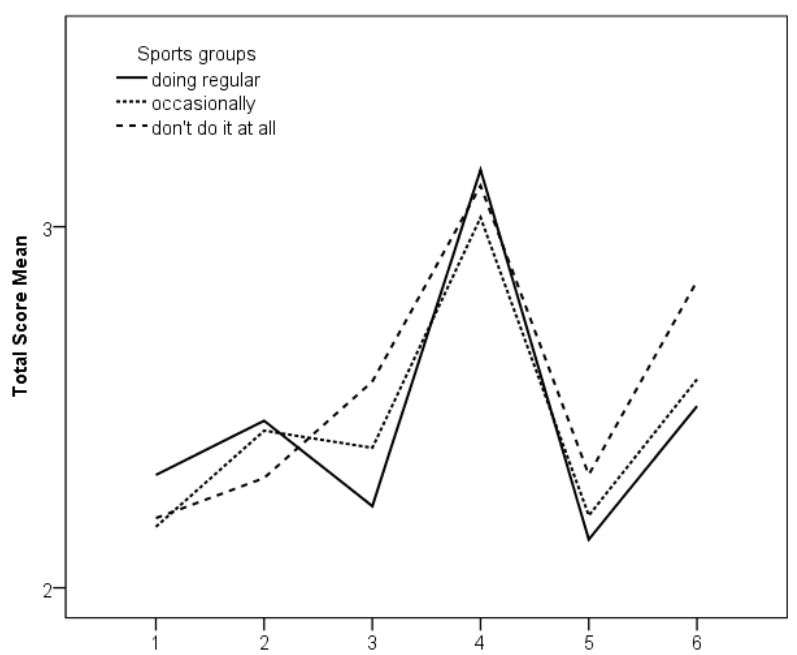

1: Honesty-Humility Domain, 2: Emotionality Domain, 3: Extraversion Domain, 4: Agreeableness Domain, 5: Conscientiousness Domain, 6: Openness to Experience Domain

Figure 2. Comparison of the total scores of the groups that were formed according to the sports status in the female group

When only the male group was considered, it was determined that the Main Effect was statistically significant; Wilks Lamda $=0.93, \mathrm{~F}(12,936)=2.88$, $\mathrm{p}<0.001$, Eta Squared $=0.04$. Statistically significant differences were detected among the groups for the Emotionality factor $(\mathrm{F}(2,473)=5.46, \mathrm{p}<0.01$, Eta Squared 
$=0.02)$, for the Extraversion factor $(\mathrm{F}(2,473)=10.19$, $\mathrm{p}<0.001$, Eta Squared $=0.04)$ and for the Openness to Experience factor $(\mathrm{F}(2,473)=4.80, \mathrm{p}<0.01$, Eta Squared $=$ $0.02)$. According to the Tukey post-hoc test result, in the Emotionality factor, those who do not do sports at all and those who do sports occasionally had lower mean scores than those who does sports regularly. In the Extraversion factor, those who do sports regularly had the lowest mean scores. There were differences among the three groups in the Openness to Experience factor; however, the group that does sports regularly had lower mean scores than the other two groups, and the group that occasionally does sports had lower average scores than the group that does not do any sports at all. No statistically significant differences were detected among the three groups for the Honesty-Humility factor $(\mathrm{F}(2,473)=2.02, \mathrm{p}>0.05$, Eta Squared $=0.01)$, for the Agreeableness factor $(\mathrm{F}(2,473)=0.29, \mathrm{p}>0.05$, Eta Squared $=0.00)$ and for the Conscientiousness factor ( $F$ $(2,473)=0.78, \mathrm{p}>0.05$, Eta Squared $=0.00)($ Table 1 , Figure 3).

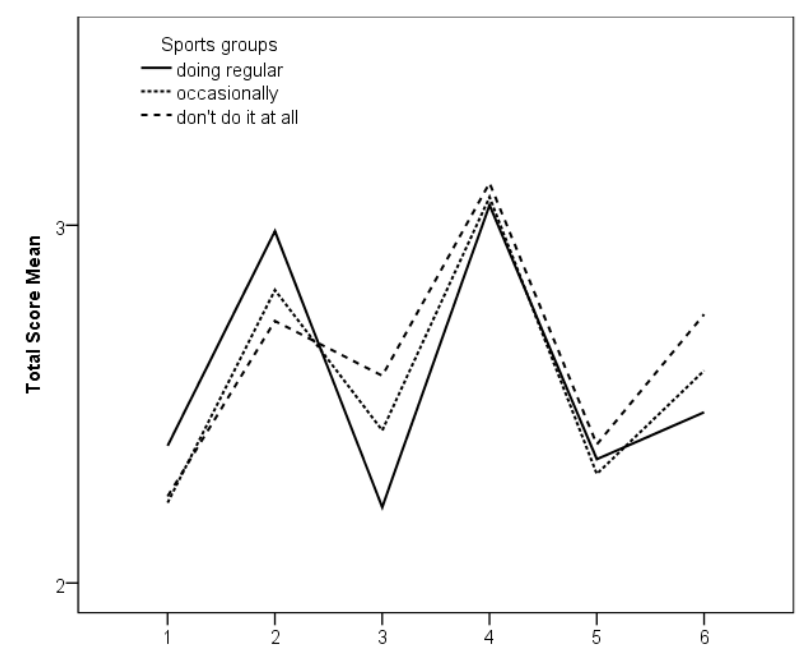

1: Honesty-Humility Domain, 2: Emotionality Domain, 3: Extraversion Domain, 4: Agreeableness Domain, 5: Conscientiousness Domain, 6: Openness to Experience Domain

Figure 3. Comparison of the total scores of the groups that were formed according to the sports status in the male group

\section{Conclusions}

There are different approaches to explain personality. Although the commonly accepted approach is the Five Factor Model, the existence of different suggestions shows that there is no consensus on this field [20]. Some of the different suggestions are: the three factor structure [21] the five factor structure $[22,23,24]$, the seven factor structure $[25,26]$ and the eight factor structure [27]. There are two different approaches that recovers the six-dimensional personality framework [4, 28]. Among these, the HEXACO model is claimed as a stronger model due to many reasons $[4,6,7,8,9,29]$. Since this model is newer than the other models, it was developed considering the problems of the previous models. As the model is relatively new and has been presented in the recent years, there are fewer studies conducted based on it compared to the former models [18]. The present study was planned with this aim, in other words, the purpose was to examine the personality profiles of the individuals who do and who do not do any sports in a Turkish-speaking participant group.

In this direction, first of all it was examined whether the individuals who do sports regularly, who do sports occasionally and who do not do any sports differed from each according to the HEXACO Personality model. It is not very clear whether doing sports affects personality traits or personality traits affect the frequency of sports participation because some personality theories emphasize the importance of the environmental factors additionally, so there is no consensus [30,31, 32, 33]. However, the main focus of this study was not the source of such differences but determining the differences. The results obtained in the present study show that there are differences among those who do sports regularly, who do sports occasionally and those who do not do any sports at all.

According to the results, differences were determined between the group that does sports regularly and the group that does not do any sports at all in the Honesty-Humility, Emotionality, Extraversion and Openness to Experience factors. The group that does sports regularly had higher scores in the Honesty-Humility and Emotionality factors, but lower scores in the Extraversion factor. The group that does not do any sports at all had lower mean scores in the Openness to Experience factor. In other words, the group that does sports regularly showed more honesty, humility and introverted characteristics than the other two groups. This group also showed more conservative characteristics compared to the group which does not do any sports at all. Similarly, the group that does sports occasionally showed more humility and introverted characteristics compared to the group that does not do any sports. These results are more meaningful when it is considered that the participants were not professional athletes.

The third question was whether the differences detected in terms of doing sports also differ in the female and male groups. According to the results, there were differences among the group who does sports regularly, the group that does sports occasionally and the group that does not do any sports at all. It was also detected in the present study that these differences were in the Emotionality, Extraversion, Conscientiousness and Openness to Experience factors in the female group; and in the Emotionality, Extraversion and Openness to Experience factors in the male group. The main difference between the female and male groups was determined in the result for Conscientiousness factor. According to the results, the female participants who do not do any sports at all had higher scores in the Conscientiousness factor than those who do sports occasionally. In other words, women who do not do sports exhibited more conscientious characteristics compared to 
those who do sports occasionally.

It was reported in previous studies that there are differences between female and male individuals in terms of personality traits $[34,35,36,37,38,39,40]$. However, it was also reported that these differences changes according to culture as well [41]. In this respect, it is understood that doing or not doing sports may reflect both social gender norms and cultural characteristics. When this logical chain is used, the results obtained are valid for the Turkish-speaking participant group. In addition, it is also understood that a similar study model may be used in different cultures and comparing the results might be more meaningful. Another recommendation that appeared in the context of the results of the present study was to repeat the comparisons made in the present study for the sub-dimensions / facets of the HEXACO Personality Inventory. Conducting a study focusing not just on the factors but the facets as well will allow researchers to investigate the relation between sports and personality in detail. This will contribute greatly to the accurate understanding for the sports-personality relation.

Following the exclusion criterion, professional athletes were not included in the study. However, this group could be included in the study. In other words, excluding this group is the limitation or missing point of the study. If professional athletes were included in the study as the fourth group, the interpretations of the results would be stronger. On the other hand, it seems that including professionals in the study will also have some drawbacks in it. For example, it is possible to foresee that the individuals dealing with individual or team sports may show differences in terms of the personality traits that will be examined in the study. In this respect, it is possible to think that sports branches might affect the results. For this reason, in such a study, it is clear that considering many variables involving sportive settings is necessary.

In this study, one of the other models that are defined as personality models could be used. It has been seen that similar studies have been conducted with the Five-Factor Personality Model, which is widely accepted. For this reason, the HEXACO Model was used. Similarly, many personality studies have been conducted on athletes. For this reason, the group who were not athletes were included in the study to show the effect of sports. The sampling that was included in this study consisted of three different groups within itself in terms of doing sports. Each group constituted reference to the other one. For this reason, another sampling that consisted of athletes was not included in the study. The study is a descriptive study explaining the personality traits of those who do and who do not do sports. For this reason, the results of the study are limited with the sampling and the personality traits included in the study. All these aforementioned aspects constitute a projection for future studies.

Including individuals who do and who do not do sports together in future studies will contribute to the understanding of the subject. In addition, including 5 and 6-Factor personality traits together will reveal the differences between the models.

When the study is evaluated in general terms, it is seen and can be said that there is a relation between doing sports and personality traits in a Turkish-speaking participant group. This result was obtained over the six-dimensional personality model using the HEXACO Personality Inventory. It is understood that the results are parallel to those reported in previous relevant studies. Lastly, although the evaluations were based on with the HEXACO model which allows cultural comparisons, detailed personality evaluations will contribute more to the understanding of the relation between doing sports and personality.

The results of this study are also important in terms of understanding the differences caused by doing and not doing sports in personality traits. In other words, this study contributes to the understanding of the differences between those who do and who do not do sports. In this context, the direct and indirect effects of doing sports on personality are understood.

\section{REFERENCES}

[1] Allport, G. W., \& Odbert, H. S. (1936). Trait-names: a psycho-lexical study. Psychological Monographs, 47(1), $1-171$.

[2] Goldberg, L. R. (1993). The structure of phenotypic personality traits. American Psychologist, 48(1), 26-34.

[3] Saucier, G., \& Goldberg, L. R. (2001). Lexical studies of indigenous personality factors: Premises, products, and prospects. Journal of Personality, 69(6), 847-879.

[4] Ashton, M. C., \& Lee, K. (2001). A theoretical basis for the major dimensions of personality. European Journal of Personality, 15(5), 327-353.

[5] Ashton, M. C., \& Lee, K. (2002). Six independent factors of personality variation: a response to Saucier. European Journal of Personality, 16(1), 63-75.

[6] Ashton, M. C., \& Lee, K. (2007). Empirical, theoretical, and practical advantages of the HEXACO model of personality structure. Personality and Social Psychology Review, 11(2), 150-166.

[7] Ashton, M. C., Lee, K., Marcus, B., \& de Vries, R. E. (2007). German lexical personality factors: Relations with the HEXACO model. European Journal of Personality, 21(1), $23-43$.

[8] Ashton, M. C., Lee, K., de Vries, R. E., Perugini, M., Gnisci, A., \& Sergi, I. (2006). The HEXACO model of personality structure and indigenous lexical personality dimensions in Italian, Dutch, and English. Journal of Research in Personality, 40(6), 851-875.

[9] Ashton, M. C., Lee, K., Perugini, M., Szarota, P., de Vries, R. E., di Blas, L., Boies, K., \& de Raad, B. (2004). A six-factor 
structure of personality-descriptive adjectives: solutions from psycholexical studies in seven languages. Journal of Personality and Social Psychology, 86(2), 356-366.

[10] Babarovic, T., \& Sverko, I. (2013). The HEXACO Personality Domains in the Croatian Sample. Društvena Istrazivanja, 22(3), 397-411.

[11] Ashton, M. C., Lee, K., \& de Vries, R. E. (2014). The HEXACO Honesty-Humility, Agreeableness, and Emotionality factors: a review of research and theory. Personality and Social Psychology Review, 18(2), 139-152.

[12] Ashton, M. C., \& Lee, K. (2009). An investigation of personality types within the HEXACO personality framework. Journal of Individual Differences, 30(4), $181-187$.

[13] Lee, K., \& Ashton, M. C. (2004). Psychometric properties of the HEXACO Personality Inventory. Multivariate Behavioral Research, 39(2), 329-358.

[14] Lee, K., \& Ashton, M. C. (2018). Psychometric properties of the HEXACO-100. Assessment, 25(5), 543-556

[15] Ashton, M. C., \& Lee, K. (2005). Honesty-humility, the Big Five, and the Five-Factor Model. Journal of Personality, 73(5), 1321-1354.

[16] Ashton, M. C., \& Lee, K. (2008a). The HEXACO model of personality structure and the importance of the $\mathrm{H}$ factor. Social and Personality Psychology Compass, 2(5), 1952-1962.

[17] Ashton, M. C., \& Lee, K. (2008b). The prediction of Honesty-Humility-related criteria by the HEXACO and Five-Factor Models of personality. Journal of Research in Personality, 42(5), 1216-1228.

[18] Tatar, A. (2018). Gözden Geçirilmiş Uzun ve Kısa Form Altı Faktörlü Kişilik Envanteri (HEXACO-PI-R) Türkçe Formunun psikometrik özelliklerinin incelenmesi. Anadolu Psikiyatri Dergisi, 19(1), 5-13.

[19] Lee, K., \& Ashton, M. C. (2006). Further assessment of the HEXACO Personality Inventory: Two new facet scales and an observer report form. Psychological Assessment, 18(2), 182-191.

[20] Block, J. (1995). A contrarian view of the five-factor approach to personality description. Psychological Bulletin, 117(2), 187-215.

[21] Barrett, P. T., Petrides, K. V., Eysenck, S. B., \& Eysenck, H. J. (1998). The Eysenck Personality Questionnaire: an examination of the factorial similarity of $\mathrm{P}, \mathrm{E}, \mathrm{N}$, and $\mathrm{L}$ across 34 countries. Personality and Individual Differences, 25(5), 805-819.

[22] Costa, P. T., \& McCrae, R. R. (1995). Primary traits of Eysenck's PEN system: three-and five-factor solutions. Journal of Personality and Social Psychology, 69(2), 308-317

[23] Goldberg, L. R. (1990). An alternative "description of personality": the big-five factor structure. Journal of Personality and Social Psychology, 59(6), 1216-1229.

[24] McCrae, R. R., \& Costa, P. T. (1987). Validation of the five-factor model of personality across instruments and observers. Journal of Personality and Social Psychology,
52(1), 81-90.

[25] Almagor, M., Tellegen, A., \& Waller, N. G. (1995). The Big Seven model: a cross-cultural replication and further exploration of the basic dimensions of natural language trait descriptors. Journal of Personality and Social Psychology, 69(2), 300-307.

[26] Saucier, G. (2002). Gone too far-or not far enough? Comments on the article by Ashton and Lee (2001). European Journal of Personality, 16(1), 55-62.

[27] Caprara, G. V., Barbaranelli, C., \& Comrey, A. L. (1992). Validation of the Comrey Personality Scales on an Italian sample. Journal of Research in Personality, 26(1), 21-31.

[28] Hogan, R., \& Hogan, J. (2007). Hogan Personality Inventory manual (3rd Ed.). Tulsa, OK: Hogan Press.

[29] Ashton, M. C., \& Lee, K. (2009). The HEXACO-60: a short measure of the major dimensions of personality. Journal of Personality Assessment, 91(4), 340-345.

[30] Boomsma, D. I., De Geus, E. J., Van Baal, G. C. M., \& Koopmans, J. R. (1999). A religious upbringing reduces the influence of genetic factors on disinhibition: evidence for interaction between genotype and environment on personality. Twin Research and Human Genetics, 2(2), $115-125$

[31] Bouchard, T. J., \& Loehlin, J. C. (2001). Genes, evolution, and personality. Behavior Genetics, 31(3), 243-273.

[32] McGue, M., Bacon, S., \& Lykken, D. T. (1993). Personality stability and change in early adulthood: A behavioral genetic analysis. Developmental Psychology, 29(1), 96-109.

[33] Riemann, R., Angleitner, A., \& Strelau, J. (1997). Genetic and environmental influences on personality: a study of twins reared together using the self- and peer report NEO-FFI scales. Journal of Personality, 65(3), 449-475.

[34] Del Giudice, M., Booth, T., \& Irwing, P. (2012). The distance between Mars and Venus: Measuring global sex differences in personality. PloS One, 7(1), e29265.

[35] Feingold, A. (1994). Gender differences in personality: a meta-analysis. Psychological Bulletin, 116(3), 429-456.

[36] Lippa, R. A. (2010). Sex differences in personality traits and gender-related occupational preferences across 53 nations: testing evolutionary and social-environmental theories. Archives of Sexual Behavior, 39(3), 619-636.

[37] Schmitt, D. P., Realo, A., Voracek, M., \& Allik, J. (2008). Why can't a man be more like a woman? Sex differences in Big Five personality traits across 55 cultures. Journal of Personality and Social Psychology, 94(1), 168-182.

[38] Vecchione, M., Alessandri, G., Barbaranelli, C., \& Caprara, G. (2012). Gender differences in the Big Five personality development: a longitudinal investigation from late adolescence to emerging adulthood. Personality and Individual Differences, 53(6), 740-746.

[39] Vianello, M., Schnabel, K., Sriram, N., \& Nosek, B. (2013). Gender differences in implicit and explicit personality traits. Personality and Individual Differences, 55(8), 994-999.

[40] Weisberg, Y. J., DeYoung, C. G., \& Hirsh, J. B. (2011). Gender differences in personality across the ten aspects of 
the Big Five. Frontiers in Psychology, 2, 1-11.

[41] Costa, P. T., Terracciano, A., \& McCrae, R. R. (2001).
Gender differences in personality traits across cultures: robust and surprising findings. Journal of Personality and Social Psychology, 81(2), 322-331. 